\title{
„Vitale“ Intralogistik - Integration von Vitaldaten in die Prozesse auf dem betrieblichen Hallenboden
}

\author{
Tim Hornung ${ }^{1}$, Matthias Bucher ${ }^{2}$, Sven Schuler ${ }^{3}$ \\ ${ }^{1}$ tim.hornung@iao.fraunhofer.de \\ ${ }^{2}$ m.bucher@viastore.com \\ ${ }^{3}$ sven.schuler@iao.fraunhofer.de \\ ${ }^{1,3}$ Fraunhofer-Institut für Arbeitswirtschaft und Organisation IAO \\ Stuttgart, Deutschland, \\ 2 viastore SOFTWARE GmbH, \\ Stuttgart, Deutschland
}

DOI: 10.14464/awic.v3i0.259

\section{ABSTRACT}

In den letzten Jahrzehnten hat die Digitalisierung alle Bereiche des Lebens durchdrungen. Internet, Computer und Smart Devices sind nicht mehr wegzudenken. In der Produktion wird die Digitalisierung mit dem Schlagwort „Industrie 4.0“ beschrieben. Ziel ist hierbei die Vernetzung von Mensch, Maschine und Produkt zu einem Internet der Dinge, Dienste und Daten. Ein weiterer Trend ist ein gesunder Lebensstil sowie eine ausgewogene Work-LifeIntegration. Besonders die Generation $Y$ strebt nach einem fitten, starken Körper und einer gesunden Ernährung. Zahlreiche Workshops und Apps unterstützen diesen Trend. Im beruflichen Umfeld gestaltet sich dieser Lebensstil jedoch schwierig. „Warum diese beiden Trends also nicht verbinden!", dachte sich das Unternehmen viastore SOFTWARE GmbH aus Stuttgart, „Arbeit, Fitness und Spaß an einem Arbeitsplatz“. Was sich für viele unvorstellbar anhört, wird im Rahmen des Forschungsprojektes „MyCPS“, in der Intralogistik umgesetzt. Ein handelsüblicher Fitness-Tracker stellt dem Warehouse-Management-System Vitaldaten wie Herzfrequenz oder ein Aktivitätsprotokoll zur Verfügung. Mithilfe dieser Daten können die auftretenden Beanspruchungen des Mitarbeiters in Relation zu seiner Tätigkeit gesetzt und somit Aspekte einer ergonomischen Arbeitsgestaltung bei gleichzeitiger Berücksichtigung der persönlichen Bedürfnisse der Mitarbeiter verwirklicht werden.

Keywords: Industrie 4.0, Work-Life-Integration, Vitaldaten, Intralogistik 


\section{WIE TRENDS DIE PRODUKTIONSARBEIT VERÄNDERN}

Seit ein paar Jahren prägen Megatrends wie Globalisierung, demografischer Wandel (Spath, 2013), Urbanisierung und Volatilität (Vernim, 2016) die Arbeit auf dem betrieblichen Hallenboden. Besonders die Digitalisierung, welche im Produktionsumfeld unter dem Titel „Industrie 4.0“ beschrieben wird, wirkt sich auf unseren Arbeitsalltag aus (Spath, 2013). In der vierten industriellen Revolution vernetzen sich intelligente, sich selbst steuernde Objekte temporär und zielgerichtet mit ihrer Umwelt und verknüpfen die reale mit der virtuellen Welt in einem Internet der Dinge, Dienste und Daten. Neue Formen von sozio-technischen Arbeitssystemen entstehen, was mit einer Veränderung der Arbeitsorganisation und -gestaltung einhergeht. Hieraus bilden sich neue Beschäftigungsformen, welche von den Mitarbeitern ein hohes Maß an zeitlicher und räumlicher Flexibilität voraussetzen sowie die Anwendung von Digitalisierung, IT und intelligenten technischen Systemen erfordern (Bauer, 2014; Spath, 2013). Neben diesen neuen Formen von sozio-technischen Arbeitssystemen rücken die individuellen Bedürfnisse der Mitarbeiter mehr in den Fokus der Arbeitsgestaltung (DGB-Index Gute Arbeit, 2017). Rahmenbedingungen wie eine flexible Gestaltung der Arbeitszeit, eine individuelle Anpassung der Arbeitssysteme an den Mitarbeiter sowie bedarfsorientierte Weiterbildungen prägen die Produktionsarbeit (Vernim, 2016). Besonders für jüngere Generationen spielt eine ausgeprägte Work-Life-Balance eine zunehmend wichtigere Rolle (Ewinger, 2016).

\section{LEBEN UND ARBEITEN IM ZEITALTER VON INDUSTRIE 4.0}

Bis Anfang der 1990iger Jahre existierte eine striktere Trennung von Berufs- und Privatleben. Erst der Begriff Work-Life-Balance beschreibt den erfolgreichen Einklang von Berufs- und Privatleben. Es soll ein ausgewogener Zustand zwischen Berufs- und Privatleben erzielt werden, damit eine dauerhafte Überlastung vermieden und eine Zufriedenheit mit der eigenen Rolle in verschiedenen Lebensbereichen ermöglicht werden kann. Beispielweise können Arbeitnehmer ihren Arbeitstag flexibel gestalten, E-Mails im Urlaub abrufen oder soziale Medien im Büro nutzen. Jedoch handelt es sich bei der Work-Life-Balance nicht ausschließlich um den Ausgleich von Beruf und Freizeit, sondern um eine Verzahnung der vorkommenden Bereiche im Leben wie Beruf, Familie und Pflege von sozialen Beziehungen, Gesundheit, Streben nach individuellen Bedürfnissen wie Anerkennung sowie spirituelle Erfüllung (Schnieder, 2013). Die Grenzen zwischen Berufs- und Privatleben sind nicht klar zu deuten und verschmelzen miteinander. Aus diesem Grund reicht der Begriff Work-Life-Balance nicht mehr aus und sollte als kontinuierlicher Prozess betrachtet werden. Neben der bisherigen Separation zwischen den Systemen ist auch eine Work-Life-Integration zu beobachten. In dieser Ausprägung verbinden sich das Berufs- und Privatleben zu einer Einheit, in welcher kein Unterschied mehr zwischen den Systemen spürbar ist (Praeg, 2017). Vor allem für jüngere Generationen soll der Beruf nicht in Konkurrenz zum Privatleben stehen. Sie sehen die Arbeitszeit als sinnvoll, erfüllend und anregend an, welche ein integraler Bestandteil des 
Lebens ist. Begriffe der ausgeprägten Leistungsorientierung und Privatleben haben somit eine besondere Bedeutung (Signium International, 2013). Generation Y oder „Digital Natives“, wie sie auch genannt werden, sind Menschen, welche zwischen Anfang der 80er und Ende der 90er des letzten Jahrtausends geboren wurden. Die Vertreter der Generation $Y$ sind mit den neusten Informations- und Kommunikationstechnologien aufgewachsen, haben maximalen Gestaltungsspielraum und Flexibilität, um ihr Leben hinsichtlich Bildungs- und Berufswahl sowie Familienplanung individuell zu gestalten als je eine andere Generation vor ihnen (Ewinger, 2016). Zusätzlich wird in dieser Generation großen Wert auf einen gesunden Lifestyle mit Sport und gesunder Ernährung gelegt (Muntschick, 2017). Viele Unternehmen haben die Bedürfnisse der Generation $Y$ bereits erkannt. Flexible Arbeitszeiten oder betriebliches Gesundheitsmanagement werden von Unternehmen für die Mitarbeiter angeboten, jedoch weitestgehend außerhalb des üblichen Arbeitsumfeldes (Ewinger, 2016). Außerdem sind diese flexiblen Arbeitsformen und neue innovative Konzepte zumeist bei Unternehmen im Bürobereich weit verbreitet und aus dem dortigen Alltag nicht mehr wegzudenken (Spath, 2013). Die viastore Software $\mathrm{GmbH}$ geht einen Schritt weiter und integriert nicht nur die sportlichen Interessen der Angestellten in ihre tägliche Arbeit, sondern auch die Work-Life-Integration in das produktive Umfeld.

\section{ARBEIT, FITNESS UND SPAß AN EINEM ARBEITSPLATZ BEI VIASTORE}

Die viastore Software $\mathrm{GmbH}$, ein Anbieter von Software für Warehouse-Management sowie vernetzte und automatisierte Materialflüsse in der Logistik und der industriellen Fertigung entwickelt im Rahmen des Forschungsprojektes „MyCPS“ ein Lösungsmodell „Warehouse 4.0" für die Intralogistik. Hierbei sollen die Mitarbeiter unter Zuhilfenahme von modernen Endgeräten (GPS und Fitness Trackern) interaktiv in die Beeinflussung ihres Arbeitsablaufs im Sinne einer besseren Produktionsplanung und -steuerung eingebunden werden. Im Forschungsprojekt „MyCPS“ (Migrationsunterstützung für die Umsetzung menschzentrierter Cyber-Physical-Systems) werden mit 14 Forschungs-, Entwicklungs,- und Industriepartnern sieben prototypische Industrie 4.0-Lösungen für die Bereiche Produktionssteuerung, Fertigung, Montage, Intralogistik und Instandhaltung umgesetzt sowie Werkzeuge und Methoden zur Gestaltung von cyber-physischen Systemen (CPS) entwickelt. Die Einbindung der Mitarbeiter bei der Gestaltung, Nutzung und Weiterentwicklung der Lösungen steht hierbei im Mittelpunkt, um Vertrauen und Akzeptanz aufzubauen.

In Laufe des Projektes wird ein adaptives, personalisierbares Arbeitssystem für operative Intralogistik-Arbeitsplätze entwickelt. Im Mittelpunkt stehen hierbei die von handelsüblichen Fitness-Trackern gesammelten Vitaldaten der Mitarbeiter. Bei der Ausübung seiner Tätigkeiten wie Fördern, Kommissioniern oder Palettieren werden neben der Herzfrequenz und der Anzahl der Schritte ebenfalls die geleistete Höhendifferenz gemessen. Die anfallenden Vitaldaten werden mit den im Warehouse-Management-System (WMS) 
hinterlegten Aufgaben verknüpft. Durch diese Verknüpfung kann der Mitarbeiter einsehen, wie sein Aktivitätsverlauf nach der erfolgreichen Durchführung seiner Tätigkeit ist. Dies macht die geleistete Arbeit für den Mitarbeiter transparent und gibt inm implizit sinnstiftendes Feedback.

Dieses automatisiert erzeugte Leistungsprotokoll stellt die Basis für die Nutzung von spielerischen Elementen dar, auch Gamification genannt. Der Mitarbeiter kann sich selbst innerhalb seines beruflichen Alltags Trainingsziele setzen, welche durch die Erfüllung seiner Tätigkeiten erreicht werden können. Finden sich mehrere Mitarbeiter in Gruppen zusammen, beispielsweise in der Frühschicht, können sich diese Gruppen auch gemeinsame Ziele setzen bzw. die erreichten Gruppenergebnisse vergleichen. Durch den entstehenden Wettbewerb werden neue Möglichkeiten der Motivationsanregung für die Mitarbeiter generiert.

Zusätzlich kann das WMS durch die Kombination der individuellen Trainingsziele, die während einer Aufgabenausführung aufgetretene Beanspruchung des Mitarbeiters sowie den zukünftigen Aufgaben, den Prozess zur Durchführung für den Mitarbeiter individuell justieren. Das WMS kann einerseits dem Mitarbeiter Tätigkeiten vorgeschlagen, die inn unterstützen, seine Ziele zu erreichen. Andererseits kann es ihn vor einer drohenden Überbeanspruchung durch z. B. Mikropausen oder einen Tätigkeitswechsel schützen. Es entsteht ein menschzentrierter Regelkreis aus ausgeführten Aufgaben, entstandener Beanspruchung, zukünftigen Aufgaben und möglichen dynamischen Aufgaben- / Prozessänderungen.

Elementare Voraussetzungen für dieses Arbeitssystem sind Transparenz, Verständnis und Vertrauen. Der entstehende Nutzen ebenso wie die Ängste vor Datenmissbrauch durch z. B. Überwachung, Leistungskontrolle sowie Jobverlust sollten klar mit den zuständigen Interessengruppen kommuniziert und ausgeräumt werden. Ein Eckpfeiler zur Schaffung dieser Voraussetzungen ist der Einsatz eines partizipativen Projektmodells zur Einführung von menschzentrierten Arbeitssystemen. Dieser Ansatz bindet alle Interessengruppen wie Mitarbeiter, Vorgesetzte, Betriebsrat, IT und Geschäftsführung bereits in den frühen Phasen des Projektes mit ein. Einen weiteren Eckpfeiler stellt die Bereitstellung eines einheitlichen Bewertungsschemas des neuen Arbeitssystems gemäß den Datenschutz-GrundverordnungsGrundsätzen dar. Dieses Schema dokumentiert, vergleichbar mit einem Produktdatenblatt, die Angaben zur Art und Inhalt der Daten, deren Verwendungszweck, Speicherdauer sowie zu technischen Maßnahmen zur Einhaltung der Angaben. 


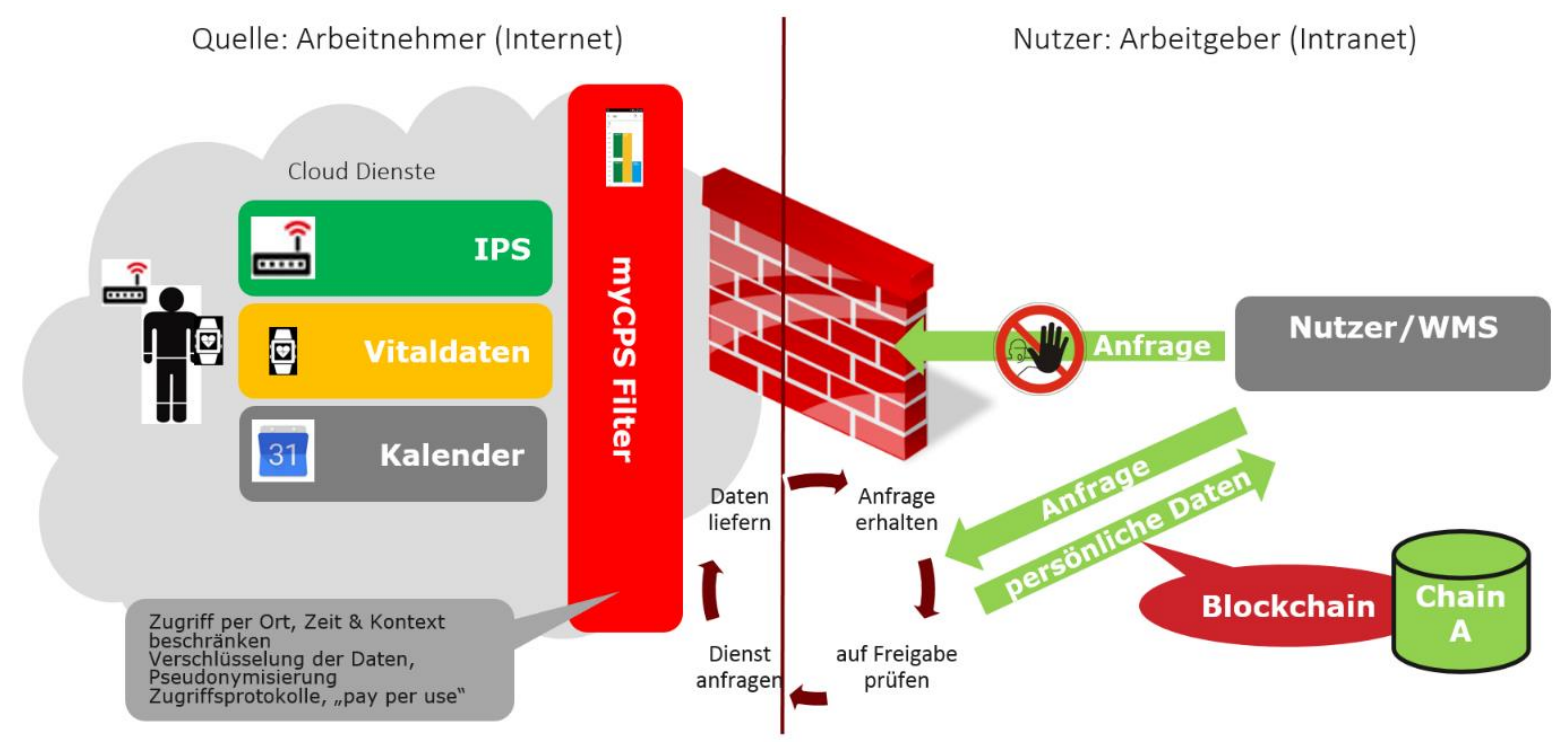

Abbildung 1: Firewall als Bindeglied zwischen Mitarbeiter und WMS System

Quelle: $\quad$ eigene Darstellung

Um die im Bewertungsschema dokumentierten Angaben technisch sicherzustellen bzw. durchzusetzen, wird ein Konzept entwickelt, um den Fluss der Mitarbeiterdaten kontrollieren zu können. Ähnlich einer Firewall verhindert der "MyCPS Filter“ den direkten, unkontrollierten und nicht protokollierten Zugriff des WMS auf die Vitaldaten des Mitarbeiters. Der Filter ist integraler Bestandteil des Arbeitssystems und stellt das Bindeglied zwischen dem Mitarbeiter und dem WMS System dar, wie in der Abbildung 1 dargestellt ist.

Die Hoheit über den Filter liegt immer beim Mitarbeiter. Es obliegt ihm selbst, den Filter über frei definierbare Regeln zu öffnen. Die Öffnung kann zeitbezogen, über Termine in einem Kalender, ortsbezogen mittels einer Bereichsmarkierung in einer Karte und nutzerkontextbezogen erfolgen. Die einzelnen Regeln können mit logischen Operatoren wie und/oder zu Sammelregeln verknüpft werden. Beispielsweise kann der Mitarbeiter einstellen, dass das WMS nur montags zwischen 8:00 und 13:00 Uhr und nur in Werkshalle 1 auf die Vitaldaten zugreifen kann.

Ein weiterer Bestandteil des Filters ist die Erzeugung eines Zugriffprotokolls. Es kann jederzeit durch den Mitarbeiter eingesehen werden und enthält chronologisch alle Zugriffsanfragen durch den Datennutzer, in diesem Fall durch das WMS. Dieses Bündel an Funktionen erlaubt es jedem Mitarbeiter eigenständig, seine informationelle Selbstbestimmung technisch und transparent gegenüber den möglichen Nutzern durchzusetzen. 


\section{FAZIT}

Aktuelle Trends wie Digitalisierung verändern die Arbeit auf dem betrieblichen Hallenboden und nehmen großen Einfluss auf die Arbeitsorganisation und -gestaltung. Besonders durch die individuellen Bedürfnisse der Mitarbeiter und Anforderungen von zeitlicher und räumlicher Flexibilität ist das Thema Work-Life-Balance stetig präsent. Berufs- und Privatleben sollten erfolgreich in Einklang gebracht werden. Jedoch vermischen sich die Grenzen zwischen Berufs- und Privatleben zunehmend miteinander. Aus diesem Grund reicht der Begriff WorkLife-Balance nicht mehr aus, da sowohl persönliche Interessen in das Berufsleben als auch berufliche Aspekte in das Privatleben integriert werden. Besonders im Büroalltag ist eine solche Work-Life-Integration weit verbreitet. Die viastore Software $\mathrm{GmbH}$ veranschaulicht in dem Forschungsprojekt „MyCPS“ wie eine mögliche Integration der persönlichen Interessen in den beruflichen Alltag im produktiven Umfeld aussehen kann. In diesem Anwendungsfall werden Vitaldaten mit Tätigkeiten in der Intralogistik verknüpft. Einerseits kann das System Tätigkeiten nach dem Fitness-Level oder der bisherigen Beanspruchung zuordnen, andererseits kann der Mitarbeiter seine Arbeitszeit als Trainingszeit sehen und persönliche Fitness-Ziele im System hinterlegen. Zusätzlich haben die Mitarbeiter die Option mit sich oder ihren Kollegen in einen spielerischen Wettbewerb zu treten. Um den Mitarbeiter vor Datenmissbrauch zu schützen, wurde neben der Datenschutz-Grundverordnung auch eine Firewall (MyCPS Filter) integriert. Diese gibt dem Mitarbeiter die Möglichkeit zu bestimmen, wann das System auf seine Vitaldaten zugreifen darf. Dieser Anwendungsfall zeigt, wie Berufs- und Privatleben auf dem betrieblichen Hallenboden ineinander integriert werden können und wie sowohl die Ziele des Mitarbeiters als auch die Ziele des Unternehmens berücksichtig werden.

\section{LITERATURVERZEICHNIS}

Bauer, W. et. al. (2014): Industrie 4.0 - Volkswirtschaftliches Potenzial für Deutschland. BITKOM Berlin, URL: https://www.bitkom.org/noindex/Publikationen/2014/Studien/Studie-Industrie-4-0Volkswirtschaftliches-Potenzial-fuer-Deutschland/Studie-Industrie-40.pdf [Stand: 10.04.2018].

DGB-Index Gute Arbeit (2017): Der Report 2017 - Wie die Beschäftigten die Arbeitsbedingungen in Deutschland beurteilen. Berlin: PrintNetwork pn / ASTOV Vertriebsgesellschaft $\mathrm{mbH}$.

Ewinger, D.; Ternes, A.; Koerbel, J.; Towers, I. (2016): Arbeitswelt im Zeitalter der Individualisierung. Wiesbaden: Springer Gabler.

Muntschick, V. (2017): Megatrend Gesundheit: Was müssen Arbeitgeber leisten? HRM Research Institute GmbH Mannheim, URL: http://hrm.at/fachartikel/megatrendgesundheit:-was-m\%C3\%BCssen-arbeitgeber-leisten\%3F--14613 [Stand: 13.04.2018].

Praeg, C.; Bauer, W. (2017): Vom Zukunftstrend zum Arbeitsalltag 4.0: Die Zukunft der Arbeit im Spannungsfeld von Work-Life-Separation und Work-Life-Integration. Wiesbaden: Springer Fachmedien. 
Schnieder, S. (2013): Work Life Balance in Unternehmen - Eine Chance im Wettbewerb um Fachkräfte. Hamburg: Diplomica Verlag GmbH.

Signium International (2013): Generation Y - Das Selbstverständnis der Manager von morgen. Signium International Düsseldorf, URL: https://www.zukunftsinstitut.de/fileadmin/user_upload/Publikationen/Auftragsstudien/s tudie_generation_y_signium.pdf [Stand: 13.04.18].

Spath, D.; Ganschar, O.; Gerlach, S.; Hämmerle, M.; Krause, T.; Schlund, S. (2013): Produktionsarbeit der Zukunft - Industrie 4.0. Stuttgart: Fraunhofer Verlag.

Vernim, S.; Wehrle, P.; Reinhart, G. (2016): Entwicklungstendenzen für die Produktionsarbeit von morgen. ZWF In Zeitschrift für wirtschaftlichen Fabrikbetrieb, Carl Hanser Verlag München, Ausgabe 9/2016, S.569-572. 